\title{
Identification of functional pathways associated with the conditional ablation of serum response factor in Dstn ${ }^{\text {corn1 }}$ mice
}

\author{
YANAN HUO $^{1}$, XIN XIE $^{1}$ and BO JIANG ${ }^{2}$ \\ ${ }^{1}$ Eye Center, Second Affiliated Hospital of Zhejiang University School of Medicine, Hangzhou, Zhejiang 310009; \\ ${ }^{2}$ Department of Ophthalmology, First Affiliated Hospital, College of Medicine, \\ Zhejiang University, Hangzhou, Zhejiang 310003, P.R. China
}

Received October 9, 2015; Accepted July 25, 2016

DOI: $10.3892 / \mathrm{mmr} .2016 .5984$

\begin{abstract}
The aim of the present study was to investigate the signaling pathways associated with functional alterations in corneal tissues following the conditional ablation of serum response factor $(S r f)$ in Dstn ${ }^{\text {corn1 }}$ mice. The gene expression profiling array GSE49688, which includes 3 samples each from the wild-type (WT), Dstn ${ }^{\text {corn1 }}$ mutant (corn1) and corn1 mice following the conditional ablation of $\operatorname{Srf}$ from the corneal epithelium [namely rescued (res)] mouse groups, was downloaded from the Gene Expression Omnibus database. The limma package was used to identify differentially expressed genes (DEGs) among the three mouse groups. DEGs were subsequently analyzed by dynamic comparison, hierarchical clustering and pathway enrichment analysis. Pathway alteration scores were also calculated in order to study the dynamic metergasis of each identified pathway. A total of 788 DEGs were identified between the corn 1 and res groups, 1,365 DEGs were identified between the corn 1 and WT groups, and 852 DEGs were identified between the res and WT groups. Among these DEGs, 228 genes were differentially expressed across all three groups, and were mainly enriched in signaling pathways involved in the regulation of the actin cytoskeleton, including the cofilin 1 (CFL1), the mitogen-activated protein kinase (MAPK) signaling pathway and focal adhesion. The dilated cardiomyopathy signaling pathway displayed the highest alteration score, and was enriched with integrin and integrin $\beta-6$ (ITGB6). In conclusion, the actin cytoskeleton regulatory pathway, MAPK and dilated cardiomyopathy signaling pathways, as well as CFL1 and ITGB6 genes, may be regulated by Srf to serve important roles in the progression of corneal disease.
\end{abstract}

Correspondence to: Dr Yanan Huo, Eye Center, Second Affiliated Hospital of Zhejiang University School of Medicine, 88 Jiefang Road, Hangzhou, Zhejiang 310009, P.R. China

E-mail:yananhuo@sina.com

Key words: corneal disease, serum response factor, differentially expressed genes, pathway enrichment analysis, pathway alteration score

\section{Introduction}

Epithelial hyperproliferation, increased angiogenesis and inflammation are biological processes associated with the pathogenesis of corneal disease, and are the primary cause of bilateral blindness worldwide $(1,2)$. In addition, a number of other disease conditions may arise as a result of abnormal epithelial cell proliferation, inflammation and angiogenesis, such as tumorigenesis and chronic inflammatory disorders $(3,4)$. As a result, investigating the molecular mechanisms underlying these conditions is of critical importance.

Dstn ${ }^{\text {corn } 1}$ mice are homozygous for a spontaneous null actin depolymerizing factor destrin (DSTN) allele, which results in an increase in serum response factor (Srf) expression. This increase in Srf production may lead to corneal abnormalities, including epithelial hyperproliferation, neovascularization and inflammation in the cornea (5). Based on these characteristics, Dstn ${ }^{\text {corn } 1}$ mice often serve as suitable in vivo models for the investigation of corneal diseases (6). Verdoni et al (1) demonstrated that conditional Srf knockout in the corneal epithelium of Dstn ${ }^{\text {corn1 }}$ mice rescues epithelial cell hyperproliferation, neovascularization and inflammatory phenotypes. In addition, previous studies have demonstrated that vascular endothelial growth factor receptor 1 (VEGFR1) was downregulated in Dstn ${ }^{\text {corn1 }}$ mice (7) and that conditional Srf knockout Dstn ${ }^{\text {corn1 }}$ mice displayed increased levels of VEGFR1 (1). The genome-wide screening of differentially expressed genes (DEGs) in the corneas of Dstn ${ }^{\text {corn1 }}$ mice has revealed that a large proportion of upregulated DEGs are targets of Srf (8). Additionally, another study by Verdoni et al (9) indicated that the B-cell receptor signaling pathway served an important role in the phenotype of Dstn ${ }^{\text {corn1 }}$ mice. Although a considerable number of studies have focused on understanding the molecular mechanisms of the Dstn ${ }^{\text {corn1 }}$ phenotype, the development of various abnormalities remains unclear.

Kawakami-Schulz et al (5) identified the gene networks that were affected by the increased expression of $\operatorname{Srf}$ in Dstn ${ }^{\text {corn } 1}$ mouse corneas. The expression profiling array GSE49688, which was provided by Kawakami-Schulz et al (5), was downloaded for analysis in the present study. The aim of the present study was to identify DEGs and perform signaling pathway analysis among three types of mice included in the GSE49688 array using various bioinformatics tools. The 
results may provide an important theoretical foundation for understanding the role of Srf in normal and abnormal corneal tissue homeostasis.

\section{Materials and methods}

Affymetrix microarray data. Data from the expression profiling array GSE49688 (5) were downloaded from the Gene Expression Omnibus database (http://www.ncbi.nlm.nih.gov/geo/). This dataset is based on the GPL16570 MoGene-2_0-st Affymetrix Mouse Gene 2.0 ST Array [transcript (gene) version] platform (Affymetrix, Inc., Santa Clara, CA, USA). In total, 9 samples are included in the datasets, with 3 samples each from the following groups: i) Wild-type (WT) mice; ii) a Dstn ${ }^{\text {corn1 }}$ mutant mouse model of corneal disease; and iii) $\operatorname{Dstn}^{\text {corn1 }}$ mutant mice following the conditional ablation of Srf from the corneal epithelium [namely the rescued (res) group].

Data preprocessing and differential expression analysis. The expression profiling probes were first annotated through annotation files. Subsequently, gene symbols were identified from annotation files, with the use of editing codes. Next, expression profiling of gene symbols was performed by Z-score normalization, as previously described (10). The linear models for microarray data (limma) version 3.28.17 (11) in R-software package (www.r-project.org) were applied to identify the DEGs among the three mouse groups. The $\log _{2}$-fold change $\left(\log _{2} \mathrm{FC}\right)$ and the false discovery rate (FDR) (12) were calculated. Genes with $\log _{2}$ FC $>1$ and an FDR $<0.05$ were considered to be DEGs and were used for subsequent analysis.

Dynamic comparison and hierarchical cluster analyses of $D E G s$. In order to verify that the three mouse groups represented three distinct states and examine their correlation at a molecular level, dynamic comparisons and unsupervised clustering analyses of DEGs were performed for the 9 samples in the GSE49688 array. DEGs between two mouse groups were determined at a time, thus obtaining three DEG groups, namely the corn1 vs. WT, res vs. corn1 and res vs. WT groups. The common DEGs among the three groups were then clustered hierarchically (13) and visualized using the TreeView program (jtreeview.sourceforge.net/) (14), and genes and samples were normalized using the median center method (15). The similarity matrix used the correlation-centered metric (16).

Pathway enrichment analysis. The Kyoto Encyclopedia of Genes and Genomes (KEGG; www.genome.ad.jp/kegg/) (17) knowledge database is a collection of online databases of genomes, enzymatic pathways and biological chemicals. In the present study, KEGG pathway enrichment analysis for the three groups of DEGs was performed. In addition, their association based on function was determined using the Database for Annotation, Visualization and Integrated Discovery (DAVID; http://david.abcc.ncifcrf.gov) gene classification tool (18). $\mathrm{P}<0.05$ was established as the threshold for the hypergeometric test.

Pathway alteration score. Quantitative scoring was performed for the potential pathways based on genes enriched in the pathway. The Euclidean distance quantitative method was used to calculate the dynamic metergasis of pathways in the corn 1 and res phenotypes compared with the WT phenotype (19). The pathway alteration score was calculated using the following formula:

$$
\mathrm{A}(\mathrm{P})=\log _{10}\left(\frac{1}{N} \sum_{i=1}^{N} \sqrt{\left(X_{\text {gens }}-Y_{\text {gens }}\right)^{2}}\right)
$$

where $A(P)$ is the alteration score of the pathway, $N$ is the number of DEGs, $X_{\text {gene }}$ is the expression value of gene in the corn 1 or res phenotypes and $\mathrm{Y}_{\text {gene }}$ is the expression value of gene in the WT phenotype group. The higher the score, the clearer the alteration degree of pathway from WT phenotype.

\section{Results}

Identification of DEGs. A pairwise comparison of genes from the WT, corn1 and res sample groups was performed in order to identify the DEGs between groups. As presented in Fig. 1A, a total of 1,365 DEGs were identified between the corn1 and WT sample groups, including 867 upregulated and 498 downregulated DEGs. Between the corn1 and res sample groups, 788 DEGs were identified, including 345 upregulated and 443 downregulated DEGs (Fig. 1B). A total of 852 DEGs were identified between the res and WT sample groups, including 593 upregulated and 259 downregulated DEGs (Fig. 1C).

Dynamic comparison and hierarchical cluster analysis of DEGs. Dynamic comparison analysis of DEGs among sample groups revealed that the number of DEGs between corn1 and WT was the greatest $(n=1,365)$. Among these, 826 genes overlapped with DEGs identified in the res vs. WT group, 763 genes overlapped with DEGs identified in the res vs. corn1 group, whereas only 6 genes were specific to the corn1 vs. WT group (Fig. 2). In addition, 228 common genes were differentially expressed across all three groups (Fig. 2). These results reflected the differences in expression between the three sample groups, with the res phenotype representing the transition state between WT and corn1 groups.

Hierarchical cluster analysis results are shown in Fig. 3. The cluster analysis demonstrated that the three groups exhibited distinct gene expression patterns, and confirmed that the res phenotype represented a transition state between WT and corn1 groups.

KEGG pathway enrichment analysis. The KEGG signaling pathways enriched by the upregulated and downregulated DEGs are shown in Table I. In the corn1 vs. WT and the res vs. WT groups, the upregulated DEGs were mainly enriched in the cytokine-cytokine receptor interaction and cell adhesion molecule signaling pathways, while the downregulated DEGs were mainly enriched in signaling pathways associated with cellular metabolism. Upregulated DEGs in the res vs. corn1 group were enriched in the retinol metabolism pathway, while downregulated DEGs were primarily enriched in the regulation of actin cytoskeleton (cofilin1 (CFL1) and CFL2) and mitogen-activated protein kinase (MAPK) signaling 
Table I. KEGG pathway enrichment analysis showing the identified DEGs among the various mouse groups.

\begin{tabular}{lll}
\hline KEGG term & Count $^{\mathrm{a}}$ & \\
\hline A, Upregulated DEGs & & \\
\hline Corn1 vs. WT & 47 & \\
mmu04060: Cytokine-cytokine receptor interaction & 21 & $9.47 \times 10^{-17}$ \\
mmu04514: CAMs & 18 & $3.32 \times 10^{-5}$ \\
mmu04670: Leukocyte transendothelial migration & 23 & $3.84 \times 10^{-5}$ \\
mmu04062: Chemokine signaling pathway & 16 & $4.15 \times 10^{-5}$ \\
mmu05322: Systemic lupus erythematosus & & $8.80 \times 10^{-5}$ \\
Res vs. WT & 41 & 14 \\
mmu04060: Cytokine-cytokine receptor interaction & 16 & $1.17 \times 10^{-17}$ \\
mmu04640: Hematopoietic cell lineage & 19 & $4.05 \times 10^{-6}$ \\
mmu04670: Leukocyte transendothelial migration & 17 & $1.00 \times 10^{-5}$ \\
mmu04062: Chemokine signaling pathway & & $3.89 \times 10^{-5}$ \\
mmu04514: CAMs & $4.86 \times 10^{-5}$ \\
Res vs. corn1 & 4 & $4.62 \times 10^{-2}$ \\
mmu00830: Retinol metabolism & & \\
\hline
\end{tabular}

\section{B, Downregulated DEGs}

\section{Corn 1 vs. WT}

$$
\text { mmu00830: Retinol metabolism }
$$

mmu00982: Drug metabolism

mmu00980: Metabolism of xenobiotics by cytochrome P450

mmu04710: Circadian rhythm

mmu00071: Fatty acid metabolism

$\begin{array}{rl}8 & 1.71 \times 10^{-4} \\ 7 & 1.93 \times 10^{-3} \\ 6 & 5.85 \times 10^{-3} \\ 3 & 2.11 \times 10^{-2} \\ 4 & 4.41 \times 10^{-2} \\ & \\ 5 & 5.03 \times 10^{-3} \\ 5 & 7.12 \times 10^{-3} \\ 3 & 7.54 \times 10^{-3} \\ 4 & 2.99 \times 10^{-2} \\ & \\ 16 & 1.43 \times 10^{-4} \\ 13 & 2.14 \times 10^{-3} \\ 14 & 8.59 \times 10^{-3} \\ 8 & 2.17 \times 10^{-2} \\ 4 & 2.42 \times 10^{-2}\end{array}$

Res vs. WT

mmu00830: Retinol metabolism

mmu00982: Drug metabolism

mmu04710: Circadian rhythm

mmu00980: Metabolism of xenobiotics by cytochrome P450

\section{C, Overlapping DEGs $(\mathrm{n}=228)$}

mmu04510: Focal adhesion

mmu04010: MAPK signaling pathway

mmu04810: Regulation of actin cytoskeleton

$\begin{array}{ll}8 & 1.64 \times 10^{-2} \\ 8 & 2.38 \times 10^{-2} \\ 7 & 3.01 \times 10^{-2}\end{array}$

${ }^{a}$ Count is the number of DEGs associated with each term. KEGG, Kyoto Encyclopedia of Genes and Genomes; DEG, differentially expressed gene; WT, wild-type; corn1, Dstn ${ }^{\text {corn } 1}$ mutant mice; res, serum response factor knockout in Dstn ${ }^{\text {cornl }}$ mutant mice; Dstn, destrin; CAMs, cell adhesion molecules; MAPK, mitogen-activated protein kinase.

pathways. In addition, the 228 overlapping DEGs among the three groups were mainly enriched in the focal adhesion, MAPK and regulation of actin cytoskeleton signaling pathways.
Pathway alteration score. The pathway alteration scores of the WT, corn 1 and res groups are shown in Fig. 4, and the 10 most altered pathways among the three groups are shown in Table II. Distance scores in Table II indicate the degree of deviation 

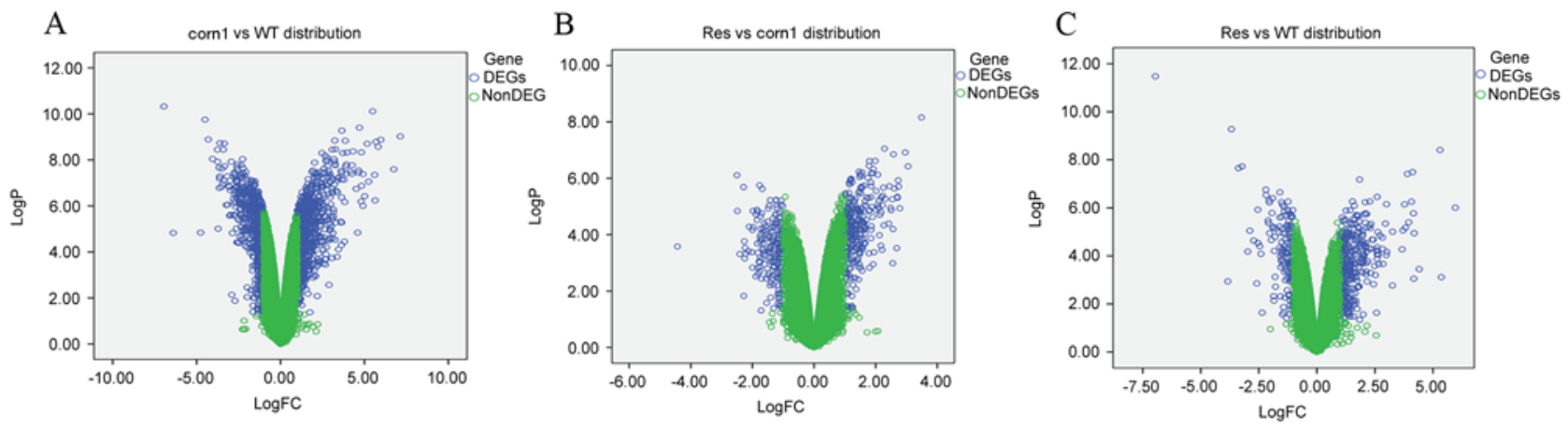

Figure 1. Volcano plots of microarray gene expression data obtained following the comparison of (A) corn1 vs. WT, (B) res vs. corn1 and (C) res vs. WT groups. The y-axis indicates the - $\log _{10}$ of P-values. DEG, differentially expressed gene; WT, wild-type; Dstn, destrin; FC, fold change; corn1, Dstn ${ }^{\text {corn } 1}$ mutant mice; res, serum response factor knockout in Dstn ${ }^{\text {corn } 1}$ mutant mice.

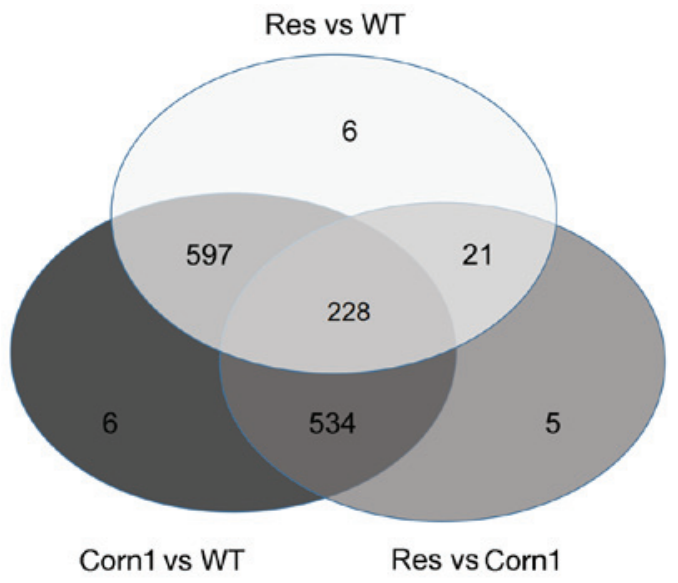

Figure 2. Venn diagram showing the differentially expressed genes among the res vs. WT, res vs. corn1 and corn1 vs. WT groups. WT, wild-type; Dstn, destrin; corn1, Dstn ${ }^{\text {corn } 1}$ mutant mice; res, serum response factor knockout in Dstn ${ }^{\text {cornl }}$ mutant mice.

between corn 1 or res groups from the WT group that is the absolute difference value between the corn and res scores. WT group served as a reference, and a high score indicated a greater degree of alteration, whereas a low score indicated that corn1 or res group mice were closer to the WT state. The pathways associated with the cardiovascular system, glycometabolism and the inflammatory response exhibited high pathway alteration scores. Of these, the dilated cardiomyopathy signaling pathway demonstrated the greatest score, and was enriched by particular DEGs, including integrin beta 6 (ITGB6).

\section{Discussion}

Srf activation has been reported to be involved in angiogenesis, the maintenance of cell hyperproliferation, inflammation and F-actin accumulation in Dstn ${ }^{\text {cornl }}$ mice (20). Notably, Srf is known to be involved in the pathogenesis of multiple types of cancer, including hepatocellular and colorectal cancer, demonstrating its potential as a disease-causing factor $(21,22)$. In the present study, 228 common genes were differentially expressed in the three groups (WT, corn1 and res), and were mainly enriched in the focal adhesion, MAPK and regulation of actin cytoskeleton signaling pathways. In addition, pathways
Table II. Pathway alteration scores for the deviation of corn1 and res groups from the WT group.

\begin{tabular}{lccc}
\hline Pathway & Corn1 & Res & Distance $^{\mathrm{a}}$ \\
\hline Dilated cardiomyopathy & -0.1 & -0.53 & 0.43 \\
Vascular smooth muscle contraction & -0.32 & -0.73 & 0.41 \\
Fructose and mannose metabolism & 0.05 & -0.31 & 0.36 \\
Galactose metabolism & 0.05 & -0.31 & 0.36 \\
MAPK signaling pathway & -0.24 & -0.59 & 0.35 \\
Regulation of actin cytoskeleton & -0.36 & -0.67 & 0.31 \\
Arachidonic acid metabolism & 0 & -0.26 & 0.26 \\
Systemic lupus erythematosus & -0.57 & -0.82 & 0.25 \\
Fatty acid metabolism & 0.05 & -0.2 & 0.25 \\
Focal adhesion & -0.34 & -0.58 & 0.24 \\
\hline
\end{tabular}

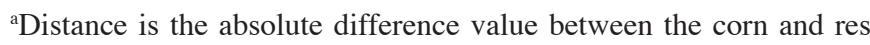
scores, with a higher value indicating a more altered pathway compared with WT. DEG, differentially expressed gene; WT, wild-type; corn1, Dstn ${ }^{\text {corn } 1}$ mutant mice; res, serum response factor knockout in Dstn ${ }^{\text {corn1 }}$ mutant mice; Dstn, destrin; MAPK, mitogen-activated protein kinase.

associated with the cardiovascular system, glycometabolism and the inflammatory response displayed high pathway alteration scores. These results may improve our understanding of the role of Srf in normal and abnormal corneal tissue homeostasis.

In the present study, the actin cytoskeleton pathway was demonstrated to be a significant pathway. In addition, overlapping DEGs in all three groups were enriched for this pathway. The actin cytoskeleton is essential for the maintenance of cell morphology and mechanical support, as well as for the regulation of diverse processes, including apoptosis, cell adhesion, cell migration and phagocytosis (23). Furthermore, the actin cytoskeleton is a critical barrier between the external environment of surrounding cells and the internal cell signaling pathways, which ultimately affects gene expression regulation (5). Ikeda et al (24) suggested that the appropriate regulation of actin dynamics is necessary for the normal maintenance of the corneal epithelium, and aberrant regulation of the actin cytoskeleton leads to epithelial cell proliferation. 

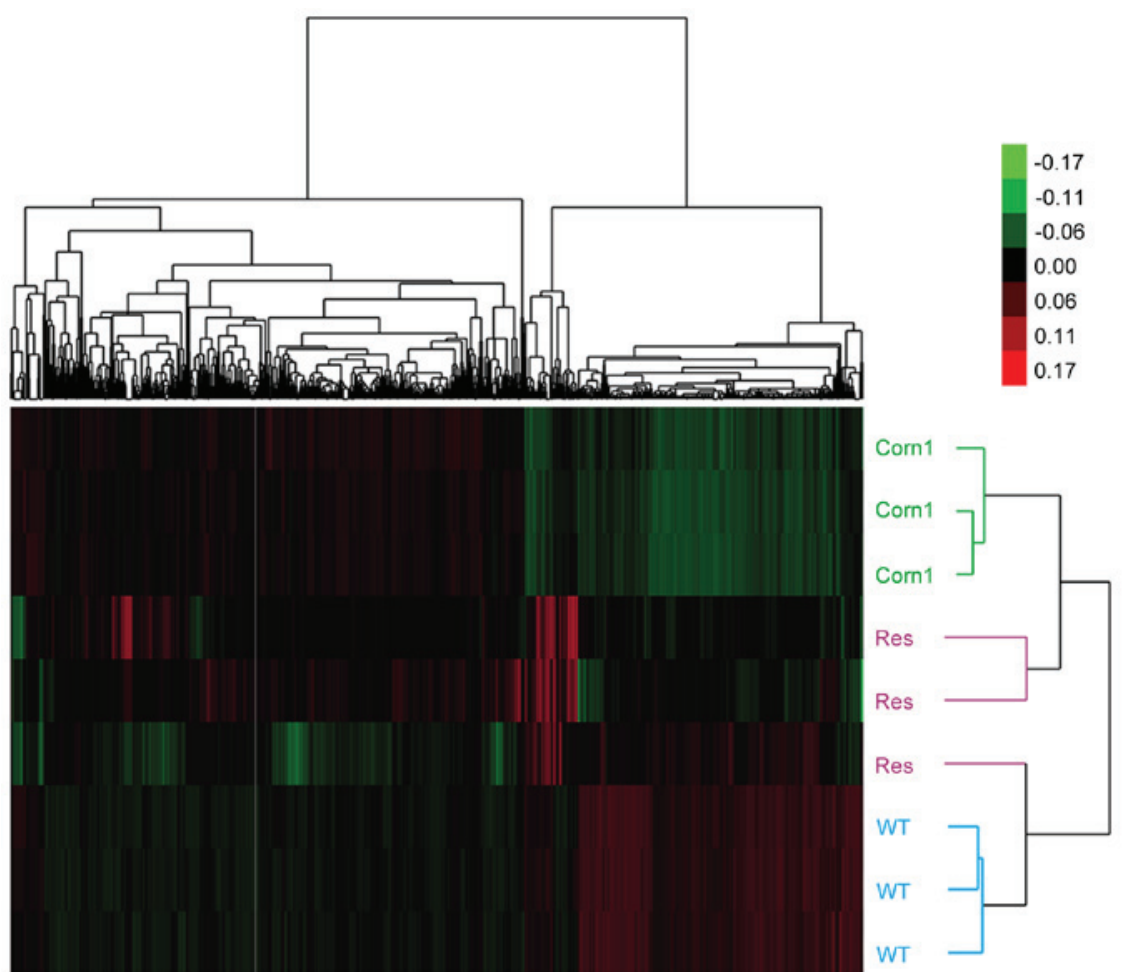

Figure 3. Hierarchical cluster analysis heat map of overlapping differentially expressed genes among the WT, corn1 and res groups. Red signals indicate increased expression and green signals indicate reduced expression. WT, wild-type; Dstn, destrin; corn1, Dstn ${ }^{\text {cornl }}$ mutant mice; res, serum response factor knockout in Dstn ${ }^{\text {cornl }}$ mutant mice.

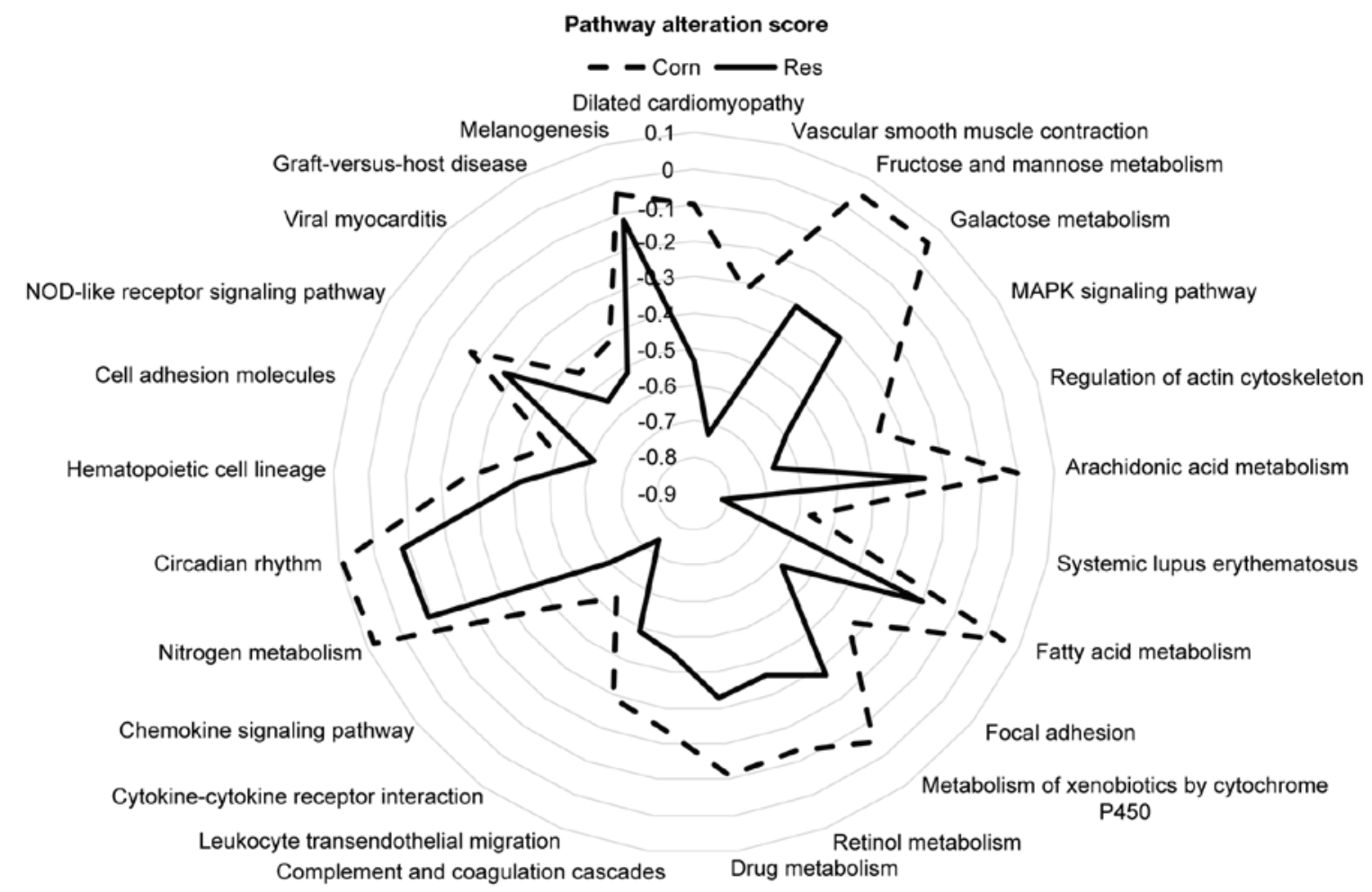

Figure 4. Pathway alteration scores in corn1 mice vs. WT, and res vs. WT groups. The dashed-line indicates the degree of deviation between corn1 and WT mice, and the filled-line indicates the degree of deviation between res mice and WT mice. Dstn, destrin; WT, wild-type; MAPK, mitogen-activated protein kinase; corn1, Dstn ${ }^{\text {cornl }}$ mutant mice; res, serum response factor knockout in Dstn ${ }^{\text {corn } 1}$ mutant mice.

Notably, Srf is an essential regulator of the actin cytoskeleton, and Srf target genes are known to be regulated by dynamic changes in the actin cytoskeleton $(8,25)$. Thus, mutant Srf-mediated regulation of the actin cytoskeleton pathway may 
serve an important role in the development of corneal disease in Dstn ${ }^{\text {corn } 1}$ mutant mice.

Previous studies have demonstrated that proteins encoded by particular Srf target genes, such as CFL1 and CFL2, serve key roles in actin treadmilling $(26,27)$. In the present study, CFL1 was enriched in the actin cytoskeleton regulatory pathway. CFL1 is a member of the actin depolymerizing factor/CFL family and is a primary regulator of actin dynamics (28). It is ubiquitously expressed and is crucial for efficient actin depolymerization (29). Notably, Ikeda et al (24) demonstrated that CFL1 and DSTN have common functions; however, compensatory mechanisms following functional loss of one actin depolymerizing factor are insufficient to restore normal actin dynamics in the cornea. Therefore, despite observing an upregulation of CFL1 in the res group compared with the corn 1 group in the present study, CFL1 may have been unable to completely regulate actin filament dynamics and compensate for the loss of DSTN.

Cell hyperproliferation, angiogenesis and inflammation are biological processes involved in the pathogenesis of corneal disease, as well as in chronic inflammatory disorders and tumorigenesis $(3,4)$. Therefore, it is possible that signaling pathways associated with cancer may be involved in the progression of corneal disease in Dstn ${ }^{\text {corn1 }}$ mutant mice. For instance, the MAPK signaling pathway was significant in the current study. Signaling to Srf occurs principally through the MAPK signaling pathway, which stimulates the expression of cell growth-promoting genes that encode proteins responsible for directly activating genes involved in cell cycle progression and growth factors $(30,31)$. Cell cycle regulation is known to be critical for the normal proliferation and development of multicellular organisms (32). The MAPK pathway is frequently activated in human cancer, which can lead to a malignant phenotype through increased cell proliferation (33). In the present study, the MAPK signaling pathway was enriched by particular downregulated DEGs in the res sample group, which indicates that $S r f$ knockout may have affected the function of this pathway. In addition, pathway alteration analysis demonstrated that the dilated cardiomyopathy signaling pathway exhibited the highest score, and ITGB6 was revealed to be enriched in this pathway.

Integrins, consisting of $\alpha$ and $\beta$ subunits, are a family of cell surface receptors that mediate cell-to-cell adhesion $(34,35)$. Notably, integrins have been reported to contribute to cell proliferation, apoptosis and the regulation of gene expression, and have been suggested to serve important roles in inflammation and tumorigenesis $(34,36)$. In the present study, ITGB6 was observed to be downregulated in the res group compared with the corn1 group, which indicated that ITGB6 may be targeted by Srf. ITGB6 is expressed specifically in epithelial cells (34); thus, epithelial hyperproliferation in the Dstn ${ }^{\text {corn } 1}$ mice may be induced by upregulation of ITGB6. Ultimately, the dilated cardiomyopathy signaling pathway and ITGB6 may serve important roles in the process of epithelial hyperproliferation and inflammation in the corneas of Dstn ${ }^{\text {corn1 }}$ mice.

In conclusion, the results of the present study provide a comprehensive bioinformatics analysis of DEGs and signaling pathways involved in the dynamic process of Dstn ${ }^{\text {corn1 }}$ mice returning to a WT-like state following the conditional ablation of $S r f$ from the corneal epithelium. The actin cytoskeleton, MAPK and dilated cardiomyopathy signaling pathways, as well as CFL1 and ITGB6 DEGs, may be regulated by Srf to serve important roles in corneal disease progression. These results may increase our understanding on the role of Srf in corneal disease tissues. However, further genetic and experimental studies with larger sample sizes are required to confirm the results of the present study, and to identify therapeutic targets for the treatment of corneal diseases.

\section{Acknowledgements}

The present study was supported by the National Natural Science Foundation of China (grant no. 81200662), the Zhejiang Provincial Natural Science Foundation of China (grant nos. LY12H12010 and LY13H120002) and the Project of Zhejiang Provincial Department of Health (grant no. 2013KYB141).

\section{References}

1. Verdoni AM, Schuster KJ, Cole BS, Ikeda A, Kao WW and Ikeda S: A pathogenic relationship between a regulator of the actin cytoskeleton and serum response factor. Genetics 186: 147-157, 2010.

2. Li Z, Cui H, Zhang L, Liu P and Bai J: Prevalence of and associated factors for corneal blindness in a rural adult population (the southern Harbin eye study). Curr Eye Res 34: 646-651, 2009.

3. Allavena P, Garlanda C, Borrello MG, Sica A and Mantovani A: Pathways connecting inflammation and cancer. Curr Opin Genet Dev 18: 3-10, 2008.

4. D'Aura Swanson C, Paniagua RT, Lindstrom TM and Robinson WH: Tyrosine kinases as targets for the treatment of rheumatoid arthritis. Nat Rev Rheumatol 5: 317-324, 2009.

5. Kawakami-Schulz SV, Verdoni AM, Sattler SG, Jessen E, Kao WW, Ikeda A and Ikeda S: Serum response factor: Positive and negative regulation of an epithelial gene expression network in the destrin mutant cornea. Physiol Genomics 46: 277-289, 2014.

6. Zhou Q, Li Y and Chen B: The effect of destrin mutations pathways on the gene expression profile. African Journal of Pharmacy and Pharmacology 6: 1746-1752, 2012.

7. Qazi Y, Wong G, Monson B, Stringham J and Ambati BK: Corneal transparency: Genesis, maintenance and dysfunction. Brain Res Bull 81: 198-210, 2010.

8. Miano JM: SRF'ing the actin cytoskeleton with no destrin. Physiol Genomics 34: 6-8, 2008.

9. Verdoni AM, Aoyama N, Ikeda A and Ikeda S: Effect of destrin mutations on the gene expression profile in vivo. Physiol Genomics 34: 9-21, 2008.

10. Murie C, Barette C, Lafanechère L and Nadon R: Control-Plate Regression (CPR) normalization for high-throughput screens with many active features. J Biomol Screen 19: 661-671, 2014.

11. Diboun I, Wernisch L, Orengo CA and Koltzenburg M: Microarray analysis after RNA amplification can detect pronounced differences in gene expression using limma. BMC Genomics 7: 252, 2006.

12. Jahani M, Nematzadeh G, Dolatabadi B, Hashemi SH and Mohammadi-Nejad G: Identification and validation of functional markers in a global rice collection by association mapping. Genome 57: 355-362, 2014.

13. Petushkova NA, Pyatnitskiy MA, Rudenko VA, Larina OV, Trifonova OP, Kisrieva JS, Samenkova NF, Kuznetsova GP, Karuzina II and Lisitsa AV: Applying of hierarchical clustering to analysis of protein patterns in the human cancer-associated liver. PLOs One 9: e103950, 2014.

14. Zhai Y, Tchieu J and Saier MH Jr: A web-based tree view (TV) program for the visualization of phylogenetic trees. J Mol Microbiol Biotechnol 4: 69-70, 2002.

15. Di Pietro C, Di Pietro V, Emmanuele G, Ferro A, Maugeri T, Modica E, Pigola G, Pulvirenti A, Purrello M, Ragusa M, et al: Anticlustal: Multiple sequence alignment by antipole clustering and linear approximate 1-median computation. Proc IEEE Comput Soc Bioinform Conf 2: 326-336, 2003.

16. Chen X: Curve-based clustering of time course gene expression data using self-organizing maps. J Bioinform Comput Biol 7: 645-661, 2009. 
17. Kanehisa M and Goto S: KEGG: Kyoto encyclopedia of genes and genomes. Nucleic Acids Res 28: 27-30, 2000.

18. Huang DW, Sherman BT, Tan Q, Collins JR, Alvord WG, Roayaei J, Stephens R, Baseler MW, Lane HC and Lempicki RA The DAVID Gene Functional Classification Tool: A nove biological module-centric algorithm to functionally analyze large gene lists. Genome Biol 8: R183, 2007.

19. Eickhoff SB,Schleicher A, Scheperjans F,Palomero-GallagherN and Zilles K: Analysis of neurotransmitter receptor distribution patterns in the cerebral cortex. Neuroimage 34: 1317-1330, 2007.

20. Verdoni AM, Schuster KJ, Cole BS, Ikeda A, Kao WW and Ikeda S: Deletion of serum response factor rescues the cornea defects caused by the loss of actin depolymerizing factor (ADF/ Destrin) in mouse. Genetics: July 6, 2010 (Epub ahead of print). doi: 0.1534/genetics.110.117309.

21. Bai S, Nasser MW, Wang B, Hsu SH, Datta J, Kutay H, Yadav A, Nuovo G, Kumar P and Ghoshal K: MicroRNA-122 inhibits tumorigenic properties of hepatocellular carcinoma cells and sensitizes these cells to sorafenib. J Biol Chem 284: 32015-32027, 2009.

22. Choi H, Kim KR, Lee JH, Park HS, Jang KY, Chung MJ, Hwang SE, Yu HC and Moon WS: Serum response factor enhances liver metastasis of colorectal carcinoma via alteration of the E-cadherin/b-catenin complex. Oncol Rep 21: 57-63, 2009.

23. Dos Remedios C, Chhabra D, Kekic M, Dedova I, Tsubakihara M, Berry D and Nosworthy N: Actin binding proteins: Regulation of cytoskeletal microfilaments. Physiol Rev 83: 433-473, 2003.

24. Ikeda S, Cunningham LA, Boggess D, Hawes N, Hobson CD, Sundberg JP, Naggert JK, Smith RS and Nishina PM: Aberrant actin cytoskeleton leads to accelerated proliferation of corneal epithelial cells in mice deficient for destrin (actin depolymerizing factor). Hum Mol Genet 12: 1029-1036, 2003.

25. Miano JM, Long X and Fujiwara K: Serum response factor: Master regulator of the actin cytoskeleton and contractile apparatus. Am J Physiol Cell Physiol 292: C70-C81, 2007.
26. Kanellos G, Zhou J, Patel H, Ridgway RA, Huels D, Gurniak CB, Sandilands E, Carragher NO, Sansom OJ, Witke W, et al: ADF and Cofilin1 control actin stress fibers, nuclear integrity, and cell survival. Cell Rep 13: 1949-1964, 2015.

27. Maciver SK and Hussey PJ: The ADF/cofilin family: Actin-remodeling proteins. Genome Biol 3: reviews3007, 2002.

28. Carlier MF, Laurent V, Santolini J, Melki R, Didry D, Xia GX, Hong Y, Chua NH and Pantaloni D: Actin depolymerizing factor (ADF/cofilin) enhances the rate of filament turnover: Implication in actin-based motility. J Cell Biol 136: 1307-1322, 1997.

29. Ichetovkin I, Grant W and Condeelis J: Cofilin produces newly polymerized actin filaments that are preferred for dendritic nucleation by the Arp2/3 complex. Curr Biol 12: 79-84, 2002.

30. Khachigian LM and Collins T: Inducible expression of Egr-1-dependent genes a paradigm of transcriptional activation in vascular endothelium. Circ Res 81: 457-461, 1997.

31. Gineitis D and Treisman R: Differential usage of signal transduction pathways defines two types of serum response factor target gene. J Biol Chem 276: 24531-24539, 2001.

32. Zhang W and Liu HT: MAPK signal pathways in the regulation of cell proliferation in mammalian cells. Cell Res 12: 9-18, 2002.

33. Sumimoto $H$, Imabayashi $F$, Iwata $T$ and Kawakami $Y$ : The BRAF-MAPK signaling pathway is essential for cancer-immune evasion in humanmelanoma cells.JExp Med 203: 1651-1656,2006.

34. Breuss JM, Gallo J, DeLisser HM, Klimanskaya IV, Folkesson HG, Pittet JF, Nishimura SL, Aldape K, Landers DV, Carpenter W, et al: Expression of the beta 6 integrin subunit in development, neoplasia and tissue repair suggests a role in epithelial remodeling. J Cell Sci 108: 2241-2251, 1995.

35. Alam N, Goel HL, Zarif MJ, Butterfield JE, Perkins HM, Sansoucy BG, Sawyer TK and Languino LR: The integrin-growth factor receptor duet. J Cell Physiol 213: 649-653, 2007.

36. Garlick DS, Li J, Sansoucy B, Wang T, Griffith L, FitzGerald T, Butterfield J, Charbonneau B, Violette SM, Weinreb PH, et al: $\alpha(\mathrm{V}) \beta(6)$ integrin expression is induced in the POET and Pten( $\mathrm{pc}-/-)$ mouse models of prostatic inflammation and prostatic adenocarcinoma. Am J Transl Res 4: 165-174, 2012. 